\title{
Correlation between Geometric Component Properties and Physical Parameters of an Aerodynamic Feeding System
}

\author{
Torge Kolditz ${ }^{1}$, Mirco Wolf and Annika Raatz ${ }^{1}$ \\ ${ }^{1}$ Institute of Assembly Technology, Leibniz University Hannover, Garbsen 30823 Germany \\ kolditz@match.uni-hannover. de
}

\begin{abstract}
In previous research, an aerodynamic feeding system was developed, which autonomously adapts to different components by using a genetic algorithm that controls the physical parameters of the system (e.g. angle of inclination, nozzle pressure). The algorithm starts with two individuals with random values, generated within the boundaries of the parameters set by the user. Due to this, the setting time - the time that passes until a satisfactory orientation rate is reached is hard to predict. The aim of this work is to identify basic interactions of geometric component properties with the physical parameters of the aerodynamic feeding system to determine in which areas of the workspace a satisfactory solution can be expected. By doing so, the initial population of the genetic algorithm can be generated based on certain geometric properties and would therefore no longer be random, presumably reducing setting time.

To identify interactions of component properties and system parameters, exemplary components were developed. They represent relevant single properties that have significant impact on the aerodynamic orientation process. These components were then fed into the aerodynamic orientation process and their behavior was documented. To identify correlations between certain geometric properties and physical parameters of the feeding system, the tests were planned and carried out using Design of Experiments methods. The results of the tests were also used to determine the direct interrelations of said properties and the suitability for aerodynamic orientation.
\end{abstract}

Keywords: Design of Experiments, Genetic Algorithm, Aerodynamic Feeding.

\section{Introduction}

In the highly automated assembly processes seen in modern production, part feeding plays a vital role. The most common part feeding system used in the industry is the vibratory bowl feeder (VBF) $[1,2]$. The essential component of a VBF is the bowl with a spiral track, which is connected to a drive unit, which creates an alternating rotational movement of the bowl using suspension springs and electromagnets [3]. This vibration is used to separate parts from the bulk at the bottom of the bowl and transport them up the spiral track, where traps are used to either reorient or reject components that are not in the desired orientation. While the bowl and the drive components have relatively low 
costs, the design of the traps and the bowl can be very expensive and timeconsuming [4]. In order to address this problem, a lot of research was done with the aim to decrease the cost for track and trap design using simulation tools [3-8]. However, even with simulation tools at hand, retooling of the orienting devices requires manual labor in the manufacturing and assembly of the specially designed bowls and traps.

To meet the demand for a highly flexible and efficient feeding technology, an aerodynamic feeding system has been developed at the Leibniz University Hannover [912]. The system uses a constant air jet to exert force on the components passing by. Using a genetic algorithm, the system is designed to parametrize itself for an optimal output rate, so that no manual labor or manufacturing is needed for retooling. The working principle of the aerodynamic feeding system and the algorithm for self-parametrization will be outlined in the following.

\section{The aerodynamic feeding system}

The aerodynamic feeding system presented and used in this work operates with only one air jet, which every component passes. In other work, systems have been presented that use multiple nozzles or air cushions to orient and transport parts [13, 14]. Fig. 1 shows the process of aerodynamic orientation in the described feeding system. It can be seen that the components behave differently, depending on the orientation they have when arriving at the nozzle. Should the workpiece arrive in the wrong orientation, it is turned over by the air jet, as can be seen in Fig. 1 a), whereas it keeps its orientation, if it already arrives in the correct orientation (Fig. 1 b)). The reason for the different behaviors of the component lies in the shape of the workpiece and the distribution of mass in the very same [13].

The shape of the workpiece influences the projected inflow area and the drag coefficient, which determine the lift force transmitted to the workpiece. If the shape of the component is not uniform over the entire length, the force transmission also changes depending on the position of the component above the nozzle. This results in a rotational impulse around the center of gravity of the workpiece. The location of the center of gravity is determined by the distribution of mass in the component. This also influences the dynamic behavior of the part when it is lifted and accelerated by the air flow. In addition to their properties, the behavior of the components in the aerodynamic orientation process is determined by the adjustment of the four physical system parameters $\alpha, \beta, v$ and $p$ seen in Fig. 1. In the exemplary process in Fig. 1, the angle of inclination $\alpha$ promotes clockwise rotation of the workpiece and impedes counterclockwise rotation, which supports the successful orientation process. The angle $\beta$ influences the force of gravity acting on the component and determines the impact of the friction between the component and the guiding plane. The nozzle pressure $p$ directly affects the magnitude of drag force acting on the workpiece. If it is set too low, the component might not rotate at all, whereas a higher pressure can lead to multiple and unpredictable rotations. Lastly, the component speed $v$ determines, how fast a workpiece passes the air jet and therefore how long the drag forces affect it. The parameter can be controlled by adjusting the speed of a conveyor located ahead of the nozzle. 
a) Workpiece arrives in the wrong position

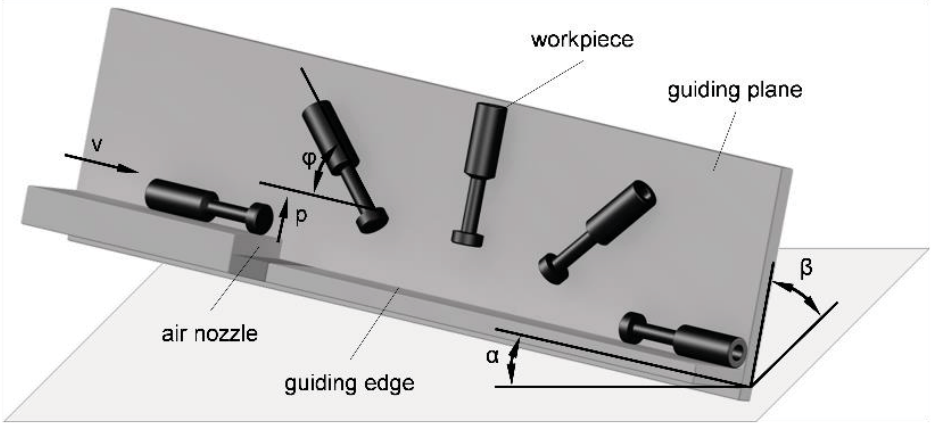

b) Workpiece arrives in the correct position

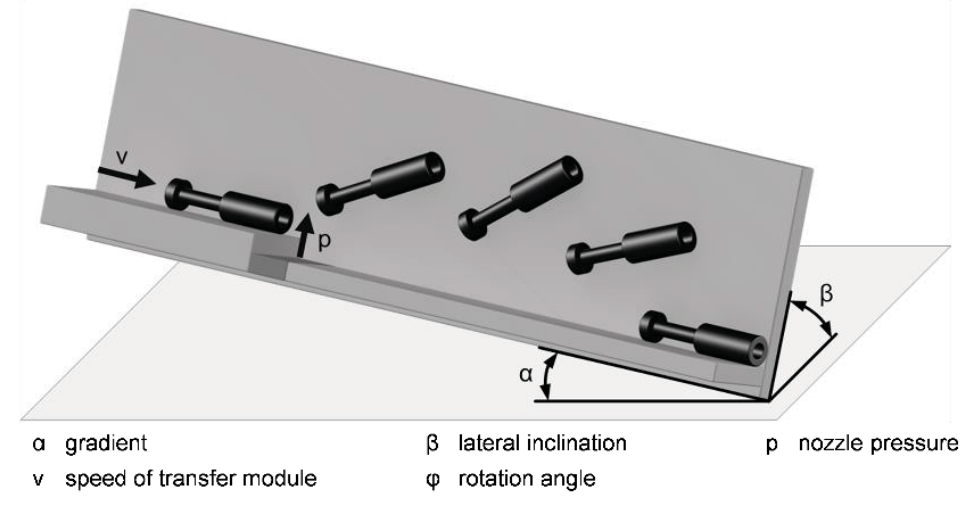

Fig. 1. Illustration of the aerodynamic orientation process [9]

In order to identify the effects and interrelations of the system parameters on the orientation process, BUSCH [15] used Design of Experiments (DoE) methods. In his work, BUSCH did not take any geometric or physical properties of the workpieces into account, because only the pneumatic plug seen in Fig. 1 was used as exemplary component. Looking at the working principle of the aerodynamic orientation process, it can be seen that certain component properties like length, diameter, shape or mass and the physical parameters of the aerodynamic feeding system (nozzle pressure, inclination, velocity) influence each other. The relevance of understanding the interaction between the component properties and the system parameters becomes apparent, when looking at the functionality of the genetic algorithm that is used to find a satisfactory set of parameters for the feeding process. The goal of the genetic algorithm (GA) is to find a set of the four physical system parameters $\alpha, \beta, v$ and $p$ that induces a satisfactory orientation rate (e.g. $>95 \%$ ). A GA was chosen for this task because the search for a good orientation rate constitutes a non-linear optimization problem with a solution space that is not necessarily continuous $[9,10,15]$. A major disadvantage regarding the convergence and predictability of the algorithm is the random initial population since there is no input correlating with the component at hand. While this assures that the whole workspace of the feeding system is searched for a solution, it also creates a huge 
solution space. The aim of this work is to determine in which areas of the workspace a satisfactory solution can be expected, depending on certain properties of the workpieces to feed by identifying basic interactions between geometric component properties and the physical system parameters $\alpha, \beta, v$ and $p$.

\section{Exemplary components}

To identify the interactions of component properties with the physical system parameters, these properties have to be defined first. The properties are divided into physical and geometrical features. Geometrical features include length, width, height, shape, symmetry and center of gravity. Relevant physical properties are mass, moment of inertia, damping properties and friction with other materials. Since it is not possible to determine the interactions of all of those properties in this work, three geometric properties are selected, that can be varied independently and determined easily. The selected properties are:

- Length

- Diameter

- Eccentricity of center of gravity

The independence of the component properties is an important factor for the DoE method because it allows a design where each experiment represents the change of only one variable without altering the others. As aforementioned, the DoE method was already used in prior work by BUSCH [15] to identify the effects and dependencies of the system parameters on the orientation of a pneumatic plug. The geometric properties were not taken into account, because only one type of plug was used. In this work, DoE is used to evaluate the effects and dependencies of geometric components properties in order to make the results more applicable to different types of workpieces.

The exemplary part used for the trials has to represent the selected properties individually, with as little interferences as possible. The simplest part that can be described with length and diameter is a cylinder. The parameters length and diameter can be varied independently. To achieve eccentricity of the center of gravity of a cylindrical part a hole from one of the front surfaces is included in the design. The eccentricity can be varied by altering the depth and diameter of the hole. At the same time, the hole does not affect the outer shape of the cylinder. Fig. 2 shows a drawing of the component, labeled sleeve in the following, with the parameters $l$ (length) and $d$ (diameter).

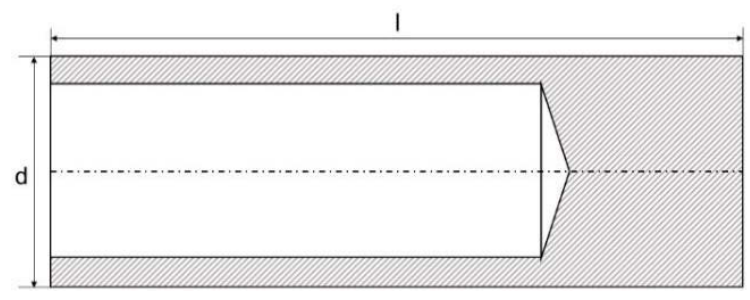

Fig. 2. Sketch of the sleeve used as exemplary component 
In order to set up a test plan, it is necessary to define the boundaries of the parameters $l$ and $d$. Suitable values for the eccentricity $\Xi$ also have to be selected. Preliminary tests show that it is very difficult to make a universal statement about the minimum and maximum values a component's property must have to be suitable for aerodynamic orientation. The reasons for this are:

- Interactions between separate component properties

- Uncontrollable behavior of the components before and after the orientation process

- Constructional characteristics of the feeding system

An example for the interaction between separate properties are the length and diameter. The boundaries of each of those properties depend, to some extent, on each other. For example, preliminary testing showed that a sleeve with a length of $12 \mathrm{~mm}$ can be oriented, if the diameter is not bigger than $6 \mathrm{~mm}$. Generally the ratio of length and diameter should be greater than two to allow aerodynamic orientation and to avoid uncontrolled behavior of the component (e.g. component tumbling down the guiding edge). Another limiting factor are the constructional characteristics of the feeding system. For example, the maximum flow and therefore the maximum impulse of the air jet is limited by the diameter of the air nozzle. The pressure of the air jet can only be increased until the critical pressure ratio $\beta$ is reached. After that, the jet is no longer usable for technical applications $[13,14]$. For air, the critical ratio is 0.528 , which limits the nozzle pressure $p$ to 0.89 bar.

Different versions of the exemplary sleeve were printed using a 3D-printer and then fed into the orientation process. A part was evaluated as generally suitable for aerodynamic feeding, if the constant air jet could reorient it. The measurements of the resulting exemplary components for the following experiments are presented in Table 1. The 3Dprinted components are shown in Fig. 3.

Table 1. Measurements of exemplary components

\begin{tabular}{cccc}
\hline Component No. & length $l$ in $\mathrm{mm}$ & diameter $d$ in $\mathrm{mm}$ & eccentricity $\Xi$ in $\%$ \\
\hline 1 & 24 & 6 & 5 \\
2 & 150 & 6 & 5 \\
3 & 24 & 12 & 5 \\
4 & 150 & 12 & 5 \\
5 & 24 & 6 & 10 \\
6 & 150 & 6 & 10 \\
7 & 24 & 12 & 10 \\
8 & 150 & 12 & 10 \\
\hline
\end{tabular}




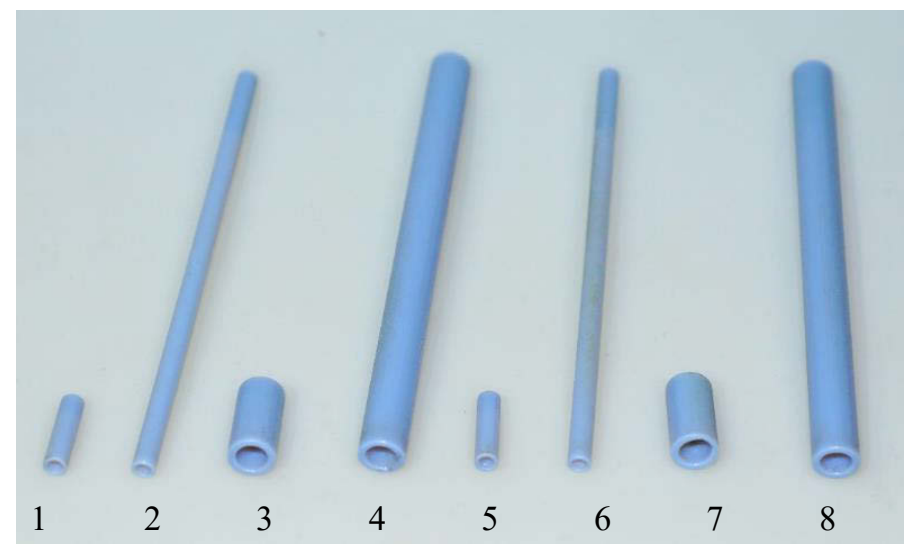

Fig. 3. 3D-printed exemplary components (sleeves) with component numbers (cf. Table 1)

\section{$4 \quad$ Interactions of component properties and system parameters}

To investigate the interaction of component properties and system parameters, an experimental plan has to be designed. In addition, the target figures of the trials have to be defined. After that, the trials are carried out and the results are evaluated.

Experimental plan. To design a proper experimental plan, it is necessary to first determine the input and output parameters of the trials. In the previous section, length, diameter and eccentricity of the components used were selected as component parameters for this work. Additionally, the physical parameters $\alpha, \beta, p$ and $v$ are defined as input parameters.

The aim of the experiments is to find out how to narrow the limits of the physical system parameters depending on the selected geometric component properties length, diameter and eccentricity in order to achieve a high orientation rate. The orientation rate is therefore defined as the only output. It is easy to quantify and has distinct limits ( $0 \%$ to $100 \%)$. Furthermore, a high orientation rate is the optimization goal of the genetic algorithm, the improvement of which is the goal of this work.

In the previous section, a full factorial test plan for the geometric properties of the components was already drawn up and corresponding values for the upper and lower limits selected through preliminary testing. In order to determine the interactions of the parameters $\alpha, \beta, p$ and $v$ and the component properties with the orientation rate, it is necessary to include the system parameters in the test plan. Table 2 shows the values of the parameters (factors) $\alpha, \beta, p$ and $v$ for each of the components.

The upper and lower factor settings $\left(x_{\min } / x_{\max }\right)$ of the physical system parameters $\alpha$, $\beta$ and $v$ are determined by the constructional boundaries of the feeding system. As mentioned before, the boundaries of the pressure $p$ are 0 bar and 0.89 bar. Since the components have different weights, as a result of the varying dimensions, the limits for this parameter cannot be generally determined for all components. If the pressure is too low, a component will not be lifted at all, if the pressure is too high, the component will spin 
multiple times, uncontrollably or even be blown out of the feeding system. To avoid this, additional preliminary tests were carried out to determine the boundaries of the pressure $p$.

Table 2. Factor setting for the physical system parameters

\begin{tabular}{ccccccccc}
\hline $\begin{array}{c}\text { Comp. } \\
\text { No. }\end{array}$ & $\begin{array}{c}\alpha_{\min } \text { in } \\
\circ\end{array}$ & $\begin{array}{c}\alpha_{\max } \text { in } \\
\circ\end{array}$ & $\begin{array}{c}\beta_{\min } \text { in } \\
\circ\end{array}$ & $\begin{array}{c}\beta_{\max } \text { in } \\
\circ\end{array}$ & $\begin{array}{c}v_{\min } \text { in } \\
\mathrm{m} / \mathrm{min}\end{array}$ & $\begin{array}{c}v_{\max } \text { in } \\
\mathrm{m} / \mathrm{min}\end{array}$ & $\begin{array}{c}p_{\min } \text { in } \\
\text { bar }\end{array}$ & $\begin{array}{c}p_{\max } \text { in } \\
\text { bar }\end{array}$ \\
\hline 1 & 20 & 24 & 40 & 50 & 55 & 75 & 0.05 & 0.1 \\
2 & 20 & 24 & 40 & 50 & 55 & 75 & 0.3 & 0.4 \\
3 & 20 & 24 & 40 & 50 & 55 & 75 & 0.5 & 0.6 \\
4 & 20 & 24 & 40 & 50 & 55 & 75 & 0.8 & 0.89 \\
5 & 20 & 24 & 40 & 50 & 55 & 75 & 0.05 & 0.1 \\
6 & 20 & 24 & 40 & 50 & 55 & 75 & 0.3 & 0.4 \\
7 & 20 & 24 & 40 & 50 & 55 & 75 & 0.5 & 0.6 \\
8 & 20 & 24 & 40 & 50 & 55 & 75 & 0.8 & 0.89 \\
\hline
\end{tabular}

The use of a full factorial test plan with four two stage factors would result in a test effort of 16 test runs per workpiece. Accordingly, a total of 128 test runs would have to be carried out. Due to the size and variety of the workpieces, the attached centrifugal feeder and the automated recirculation system cannot be used. Therefore, the components have to be applied to the conveyor (parameter $v$ ) manually. In order to reduce the testing effort, the full factorial test plan is reduced to a screening plan presented in Table 3. In order to reduce the influence of environmental conditions (temperature, humidity), the screening plan was run twice in the interval of two weeks. The order of the individual test runs ( 8 runs per workpiece) was randomized. For each test run, 100 workpieces are fed into the orientation process in alternating initial orientation. This means that if no reorientation takes place, the orientation rate is $50 \%$. If all workpieces that arrive at the nozzle in wrong orientation are reoriented, but none of the workpieces arriving at right orientation are reoriented, the orientation rate is $100 \%$.

Table 3. Screening test plan for each component

\begin{tabular}{ccccc}
\hline Test run No. & $\alpha$ & $\beta$ & $v$ & $p$ \\
\hline 1 & - & - & - & - \\
2 & + & - & - & + \\
3 & - & + & - & + \\
4 & + & + & - & - \\
5 & - & - & + & + \\
6 & + & - & + & - \\
7 & - & + & + & - \\
8 & + & + & + & + \\
\hline
\end{tabular}

Results. The results of the two screening trials show a high consistency regarding the orientation rates measured for each parameter configuration. Since the presentation of the results of all experiments would go beyond the scope of this work, the analysis of 
the test results is presented in the following. In order to determine the influence of a system parameter on the orientation rate $O$, the main effects of each parameter have to be calculated. Table 4 shows the main effects of all components together with the specific properties of the components. For example, the main effect $E_{v}$ for the second component indicates that increasing the velocity $v$ from 55 to $75 \mathrm{~m} / \mathrm{min}$ is associated with an average increase of the orientation rate of $15.9 \%$. To calculate the effect of the parameter $v$ for the first component, the results of the 16 test runs (two screenings with eight test runs each) done with the component are split in two halves, one representing the test runs with high velocity $v$ and the other representing those with a low velocity. The effect is then calculated by subtracting the mean orientation rate of the half with lower velocity from the mean orientation rate of the half with higher velocity (equation 1). In addition to the main effects, secondary effects - interactions between the factors - play a role, but will not be discussed in more detail here.

$$
\mathrm{E}_{\mathrm{v}, \text { comp } 1}=\frac{\sum \mathrm{O}_{\mathrm{v}+}}{8}-\frac{\sum \mathrm{O}_{\mathrm{v}-}}{8}=15.9 \%
$$

Table 4. Main effects of the physical parameters on the orientation rate

\begin{tabular}{cccccccc}
\hline Comp. No. & $l$ in $\mathrm{mm}$ & $d$ in $\mathrm{mm}$ & $\Xi$ in $\%$ & $E_{\alpha}$ in $\%$ & $E_{\beta}$ in $\%$ & $E_{v}$ in $\%$ & $E_{p}$ in $\%$ \\
\hline 1 & 24 & 6 & 5 & 6.6 & 3.6 & 4.9 & 0.4 \\
2 & 150 & 6 & 5 & 11.4 & -5.6 & 15.9 & -3.6 \\
3 & 24 & 12 & 5 & -7.5 & 10.3 & -4.3 & -1.5 \\
4 & 150 & 12 & 5 & -11.4 & -10.4 & 1.9 & 8.4 \\
5 & 24 & 6 & 10 & 1.1 & 1.4 & 4.6 & -3.1 \\
6 & 150 & 6 & 10 & 14.0 & -14.3 & 7.3 & -8.0 \\
7 & 24 & 12 & 10 & -8.1 & 9.6 & -4.1 & -2.9 \\
8 & 150 & 12 & 10 & -2.3 & -15.8 & -23.8 & 10.3 \\
\hline
\end{tabular}

Derived from the analysis of the main effects and the secondary effects, recommendations for the selection of the system parameters can be made. These are given in Table 5. In the table, a "+" represents a high value of the component property or system parameter and a "-"represents a low value. For example, the combination " $l-$-", " $d+$ " and " $\Xi+$ " represents a component with a small length, a high diameter and a high eccentricity of the center of gravity. For such a component, a low inclination angle $\alpha$, a high inclination angle $\beta$ and a high velocity $v$ should be selected to achieve higher orientation rates. It is expected that by narrowing the range of those parameters in an area, where high orientation rates are expected to be more likely, the setting time of the system can be reduced. The pressure $p$ has to be considered with caution, as the upper and lower limits of the pressure are not constant between the different components in contrast to the limits of the other three system parameters. 
Table 5. Recommendation for the selection of system parameters dependent on component properties

\begin{tabular}{|c|c|c|c|c|c|c|}
\hline$l$ & $d$ & $\Xi$ & $\alpha$ & $\beta$ & $v$ & $p$ \\
\hline \multirow{4}{*}{-} & \multirow{2}{*}{-} & - & \multirow{2}{*}{+} & \multirow{4}{*}{+} & \multirow{2}{*}{+} & + \\
\hline & & + & & & & - \\
\hline & \multirow{2}{*}{+} & - & \multirow{2}{*}{-} & & - & \\
\hline & & + & & & + & - \\
\hline \multirow{4}{*}{+} & \multirow{2}{*}{-} & - & \multirow{2}{*}{+} & \multirow{4}{*}{-} & \multirow{2}{*}{+} & \multirow{2}{*}{-} \\
\hline & & + & & & & \\
\hline & \multirow{2}{*}{+} & - & \multirow{2}{*}{-} & & \multirow{2}{*}{ - } & \multirow{2}{*}{+} \\
\hline & & + & & & & \\
\hline
\end{tabular}

\section{Interaction of component properties and orientation rate}

In the previous section, interactions between component properties and system parameters were investigated. In the following section, the results of the tests carried out are further analyzed to find out how the properties of the components and the orientation rate are related. The results will be used to help predict the suitability of a workpiece for aerodynamic orientation.

In order to evaluate the influence of the component properties length, diameter and eccentricity, again, a quality characteristic must be selected. As described in section 4, the orientation rate is the most suitable characteristic to determine the suitability for aerodynamic orientation. In the tests, orientation rates were determined for 64 different combinations of component properties and parameter sets. From the results of these tests, different variants of the quality characteristic orientation rate $O$ can be derived. On the one hand, the mean value of the orientation rate for each exemplary component $O_{\text {mean }}$ is selected as quality characteristic. The value indicates the average orientation rate that is achieved with one component. On the other hand, the maximum value, which indicates the highest orientation rate accomplished with an exemplary component $O_{\max }$ is selected. The values are given in Table 6 .

Table 6. Mean and maximum orientation rate of 16 test runs for each component

\begin{tabular}{cccccc}
\hline Comp. No. & $l$ in $\mathrm{mm}$ & $d$ in $\mathrm{mm}$ & $\Xi$ in $\%$ & $O_{\operatorname{mean}}$ in $\%$ & $O_{\max }$ in $\%$ \\
\hline 1 & 24 & 6 & 5 & 57,6 & 66,0 \\
2 & 150 & 6 & 5 & 65,3 & 92,0 \\
3 & 24 & 12 & 5 & 43,0 & 57,5 \\
4 & 150 & 12 & 5 & 61,1 & 94,5 \\
5 & 24 & 6 & 10 & 73,3 & 79,0 \\
6 & 150 & 6 & 10 & 56,6 & 90,0 \\
7 & 24 & 12 & 10 & 43,8 & 57,0 \\
8 & 150 & 12 & 10 & 66,4 & 98,0 \\
\hline
\end{tabular}

The values in Table 6 are used to determine the effects of the individual component properties on the orientation rate. To do so, the main effects of parameters and their 
interrelations are calculated analogously to section 4 (cf. equation 1). These factors are calculated using the mean orientation rate of all test runs with one component as quality criteria on the one hand and the maximum orientation reached with a component on the other hand. The results are illustrated in Fig. 4, sorted by size of the effects. It can be seen, that the factors have similar values, regardless of whether they are calculated from the mean or maximum values of the orientation rate in Table 6.

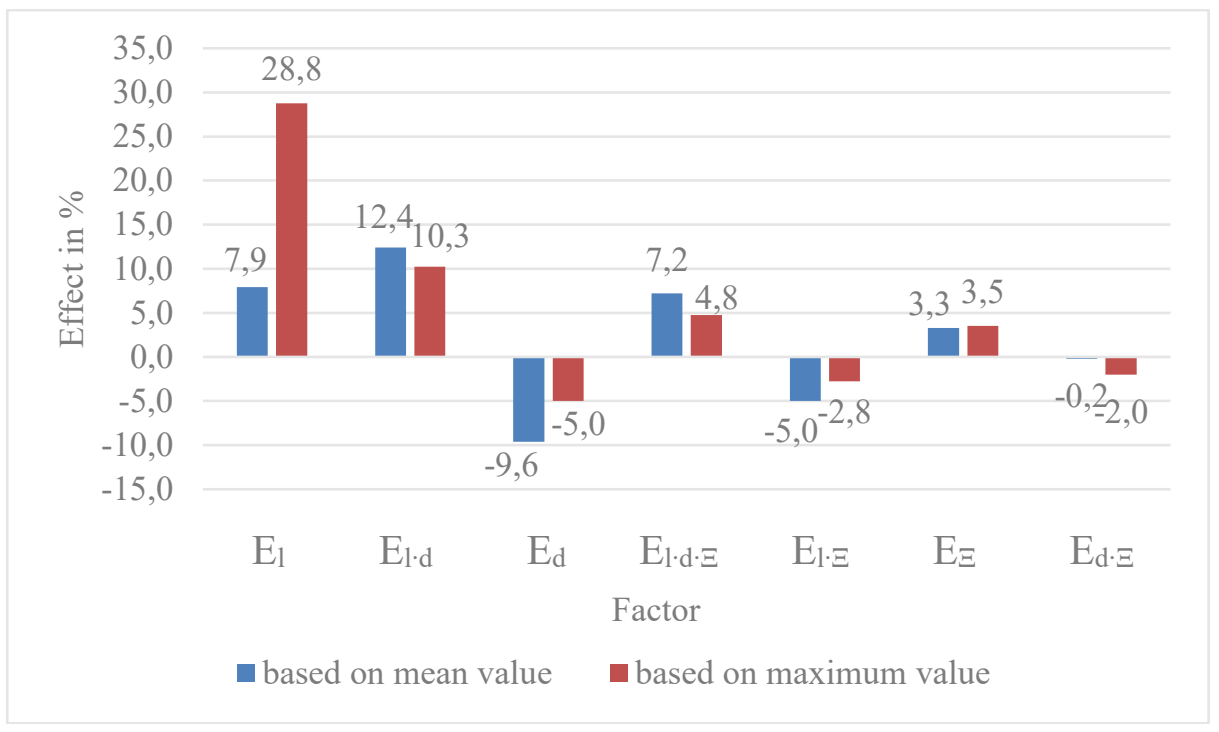

Fig. 4. Visualization of the effects of component properties and their interrelations (factors) on the orientation rate sorted by size

The results indicate that the length of a component has a large positive impact on the orientation rate. With an effect of $7.9 \%$ calculated from the average orientation rate and $28.8 \%$ calculated from the maximum orientation rate, the length has the largest effect of the three properties investigated in this work. Therefore, it can be concluded that longer components are potentially more suitable for aerodynamic orientation than shorter components. This may be because, on the one hand, a longer component is in the air jet for a longer period of time, when moving at the same velocity as a smaller component and is therefore more strongly influenced by the air jet. On the other hand, the lever with which the lifting force of the air jet rotates the component around the center of gravity becomes longer. It has been observed in the preliminary tests that longer components tend to have a more stable movement behavior on the guiding plane and edge.

The second largest effect is the two-factor interaction of length and diameter, which is positive. That indicates that components with a very large or a very small length to diameter ratio are less suitable for the orientation process. The ratio of the components used in the test runs varies from $l / d=2$ to $l / d=25$. From the results illustrated in Fig. 4 it can be deduced that both extreme characteristics achieve a lower orientation rate in comparison to the components with ratios like $l / d=4$ or $l / d=12.5$. 
The effect of the diameter is negative, which indicates that the orientation rate diminishes as the diameter of the component increases. This could already be observed in the preliminary testing and is attributed to the Bernoulli Effect, which reduces the lifting force applied to a component. When the sleeve passes over the nozzle, it creates a gap between itself and the nozzle guiding edge. Therefore, the flow path of the air jet is narrowed which results in a higher velocity of the fluid. This, according to Bernoulli's principle, leads to a decrease of pressure between the sleeve and the guiding edge ultimately resulting in the part being dragged down [14-16]. This effect becomes stronger with increasing component diameter.

The eccentricity of the center of gravity has a positive influence on the orientation rate. This has already been investigated by LORENZ [13] and is confirmed by the tests carried out for this work. An interesting observation concerns the interaction of eccentricity and length, which is negative. From this it can be concluded that an increase of the eccentricity has a higher positive impact on the orientation rate for short components than for long components. The two-factor interaction of eccentricity and diameter is very small and therefore not further discussed.

The three-factor interaction of length, diameter and eccentricity is positive. The Interpretation of this interaction is difficult because for some combinations of geometric properties it contradicts the other effects. For example, according to the three-factorinteraction, a long component with a high eccentricity of the center of gravity must have a high diameter to reach a high orientation rate. This contradicts the clearly negative effect of the diameter. At the same time, the three-factor interaction can be used to determine what a suitable component should look like. It is difficult to make a generalized statement about the geometric properties of a suitable workpiece, since the suitability is also influenced by factors not considered in this work, like nozzle diameter and shape. Yet, the results obtained by the analysis of the test data indicate that workpieces with a higher length (cf. $E_{l}$ ) and eccentricity (cf. $E_{\Xi}$ ) as well as a medium sized diameter (cf. $E_{l \cdot d}$ and $E_{l \cdot d \cdot \Xi)}$ are more suitable for aerodynamic orientation in the sense that a high orientation rate can be reached at different parameter configurations.

\section{Conclusion and Outlook}

The aim of this work was to determine the interaction between component properties and the setting of the physical parameters of the aerodynamic feeding system. In addition, it was to be found out how the change of individual component properties affects the suitability for aerodynamic orientation.

In order to achieve these objectives, relevant component properties were defined in a first step. By conducting preliminary tests, suitable sample components were designed and subsequently manufactured using a $3 \mathrm{D}$ printer. Based on eight sample component geometries, a screening test plan was drawn up in order to be able to represent the influencing factors well. A total of 128 test runs were carried out with 16 test runs per component geometry. In each test run, 100 components were fed to the aerodynamic orientation process and the resulting orientation rate was measured. 
By evaluating the test results it was possible to create a matrix which makes it possible to generate recommendations for the setting of the physical system parameters $\alpha$, $\beta, v$ and $p$, depending on given component properties such as length, diameter and eccentricity of the center of gravity (cf. Table 5). The findings can be used to narrow the range of the system parameters in order to reduce the search space and therefore the setting time of the genetic algorithm. Furthermore, positive and negative effects of certain component properties on the orientation rate and thus on the general suitability for aerodynamic orientation could also be determined (cf. Fig. 4). A higher suitability means that a high orientation rate can be achieved easier, which in return also decreases the setting time of the genetic algorithm.

The interactions and correlations derived from the tests carried out in this work are based on the aforementioned set of 128 test runs (two screenings with 64 test runs each). The goal of future work is to increase the accuracy of the recommendation matrix by repeating the test to reduce the influence of statistical errors. Also, the tests should be carried out using a different test plan. Using the screening test plan, it was assumed, that the interrelations are linear in between the boundary values. One reason for using a genetic algorithm for setting the system parameters is the non-linearity of the optimization problem. Therefore, to better model the interrelations in the entire workspace of the aerodynamic feeding system, a new test plan with a Central-Composite-Design (CCD) will be generated and carried out. The results of the CCD test plan will also be used to refine and validate the interrelation of component properties and suitability for aerodynamic orientation as shown in section 5 .

Another objective of future work will be to investigate if the results generated with the exemplary components in this work are applicable to components of entirely different geometry. The sleeves used in this work were designed to only represent the effect of an eccentric center of gravity, length and diameter on the orientation process. With the cylindrical outer contour, a very simple geometry was chosen. The aim of future work must therefore also be to reproduce the interrelationships of the component properties with the orientation process for more complex components. Two further component geometries have already been developed for this purpose and can be seen in Fig. 5 . With those components, the success of the aerodynamic orientation process is not only based on the eccentricity of the center of gravity but also on the varying projected inflow surface of the components.

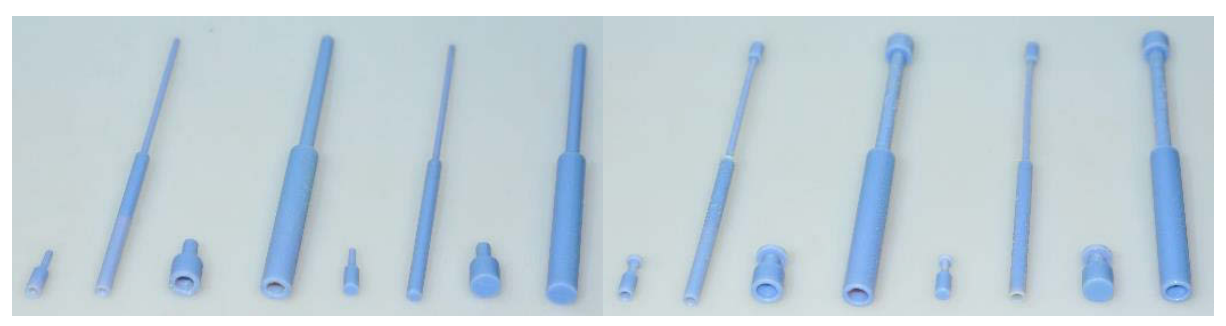

Fig. 5. 3D-printed exemplary components for further testing 


\section{$7 \quad$ Acknowledgements}

The results presented in this paper were obtained within the project "Model-based increase of the flexibility and robustness of an aerodynamic part feeding system for highperformance assembly (project number: 243351293). The authors would like to thank the German Research Foundation (DFG) for their financial support of this project.

\section{References}

1. Boothroyd, G.: Assembly Automation and Product Design. 2nd edn. Talyor \& Francis Group, Boca Raton (2005).

2. Rockland, M.: Flexibilisierung der automatischen Teilebereitstellung in Montageanlagen. Springer, Berlin Heidelberg (1995).

3. Stocker, C., Hell, M., Reisch, R., Reinhart, G.: Automated Generation of Orienting Devices for Vibratory Bowl Feeders. In: Proceedings of the 2017 IEEE International Conference on Industrial Engineering and Engineering Management (IEEM), pp. 1586-1590. IEEE, Singapore (2017).

4. Mathiesen, S., Ellekilde, L.-P.: Configuration and Validation of Dynamic Simulation for Design of Vibratory Bowl Feeders. In: Proceedings of the 12th IEEE International Conference on Control \& Automation (ICCA), pp. 485-492. IEEE, Kathmandu, Nepal (2016).

5. Hofmann, D., Huang, H., Reinhart, G.: Automated Shape Optimization of Orienting Devices for Vibratory Bowl Feeders. In: Journal of Manufacturing Science and Engineering Vol. 135 (5): 051017, ASME (2013).

6. Hansson, M. N., Mathiesen, S., Ellekilde, L.-P., Madsen, O.: Configuration System for Simulation Based Design of Vibratory Bowl Feeders. In: Proceedings of the 2016 IEEE International Conference on Simulation, Modeling and Programming for Autonomous Robots, pp. 147-154. IEEE, San Francisco, USA (2016).

7. Hofmann, D., Reinhart, G.: Simulationsgestützte Auslegungsmethode für Ordnungsschikanen. In: ZWF Zeitschrift für wirtschaftlichen Fabrikbetrieb 108 (3), pp. 148-153. Carl Hanser Verlag, München (2013).

8. Goemanns, O. C., van der Stappen, A. F.: On the design of traps for feeding 3D parts on vibratory tracks. In: Robotica vol. 26, pp. 537-550. Cambridge University Press, Cambridge (2008).

9. Busch, J.; Blankemeyer, S.; Raatz, A.; Nyhuis, P.: Implementation and Testing of a Genetic Algorithm for a Self-learning and Automated Parameterization of an Aerodynamic Feeding System. In: Procedia CIRP 44, pp. 79-84. Elsevier (2016).

10. Busch, J.; Knüppel, K.: Development of a Self-Learning, Automatic Parameterisation of an Aerodynamic Part Feeding System. In: Advanced Materials Research vol. 769, pp. 34-41. (2013).

11. Fleischer, J.; Herder, S.; Leberle, U.: Automated supply of micro parts based on the micro slide conveying principle. In: CIRP Annals 60 (1), pp. 13-16. (2011).

12. Frädrich, T.; Pachow-Frauenhofer, J.; Torsten, F.; Nyhuis, P.: Aerodynamic feeding systems. An example for changeable technology. In Assembly Automation 31 (1), pp. 47-52. (2011).

13. Lorenz, B.-M.: Aerodynamische Zuführtechnik. In: Fortschritt-Berichte VDI; Reihe 2, Fertigungstechnik, 524. VDI-Verlag, Düsseldorf (1999). 
14. Rybarczyk, A.: Auslegung aerodynamischer Zuführverfahren. In: Berichte aus dem IFA 1, pp. 1-124. Produktionstechnisches Zentrum Hannover, Garbsen (2004).

15. Busch, J.: Entwicklung einer intelligenten aerodynamischen Zuführanlage für die Hochleistungsmontage. In: Berichte aus dem IFA 7/2016. PZH Verlag, Garbsen (2016).

16. Chattot, J. J.; Hafez, M. M.: Theoretical and Applied Aerodynamics. Springer, Dodrecht (2015)

Open Access This chapter is licensed under the terms of the Creative Commons Attribution 4.0 International License (http://creativecommons.org/licenses/by/4.0/), which permits use, sharing, adaptation, distribution and reproduction in any medium or format, as long as you give appropriate credit to the original author(s) and the source, provide a link to the Creative Commons license and indicate if changes were made.

The images or other third party material in this chapter are included in the chapter's Creative Commons license, unless indicated otherwise in a credit line to the material. If material is not included in the chapter's Creative Commons license and your intended use is not permitted by statutory regulation or exceeds the permitted use, you will need to obtain permission directly from the copyright holder. 